\title{
Preparation and Research on the Electromagnetic Wave Absorbing Coating with Co-Ferrite and Carbonyl Iron Particles
}

\author{
Xinwei $\mathrm{Ji}^{1,2}$, Ming $\mathrm{Lu}^{1}$, Feng $\mathrm{Ye}^{1} \&$ Qian Zhou ${ }^{1}$ \\ ${ }^{1}$ College of Field Engineering, PLA University of Science and Technology, Nanjing, China \\ ${ }^{2} 3$ shi, Mail-box 2862\#, No.11 Anning Zhuang Road, Haidian District, Beijing, China \\ Correspondence: Ming Lu, College of Field Engineering, PLA University of Science and Technology, No.1 Hai \\ Fu Xiang Street, Bai Xia District, Nanjing 210007, China. Tel: 86-187-0156-4183. E-mail: jxw0127@gmail.com
}

\author{
Received: December 15, 2012 Accepted: January 7, 2013 Online Published: January 28, 2013 \\ doi:10.5539/jmsr.v2n2p35 \\ URL: http://dx.doi.org/10.5539/jmsr.v2n2p35
}

\begin{abstract}
Microwave absorbing coatings composed of Co-Ferrite particles and carbonyl iron particles (CIP) as absorbent and aliphatic polyurethane resin as matrix were fabricated, and their electromagnetic and microwave absorbing properties could be tuned by changing the weight fraction of Co-ferrite at 4-18 GHz. The result of reflectivity indicates that the coating with Co-ferrite particles have excellent absorption in the range of $12 \mathrm{GHz}-18 \mathrm{GHz}$ with reflection loss below $-10 \mathrm{~dB}$. The absorption with carbonyl iron particles is less than $-8 \mathrm{~dB}$ in the range of 4 GH-8 GHz. The hybrid of two particles was used to fabricate the coating in order to broadening the effective bandwidths. The reflectivity results demonstrated that the maximum absorption of the coating with hybrid particles is less than that containing only one kind particle. But the effective bandwidths $(<-8 \mathrm{~dB})$ increases to $13 \mathrm{GHz}$. This research work conludes that having both Co-Ferrite particles and carbonyl iron particles as absorbent is an effective way to broaden the bandwidths of absorption.
\end{abstract}

Keywords: Co-ferrite particles, carbonyl iron particles, electromagnetic parameters, reflectivity, electromagnetic wave absorbing coating

\section{Introduction}

Radar absorbing materials (RAMs) for commercial and military application have been started since 1930s. But still it is a hot topic of great interest in research field (Bhattacharya et al., 2012). The used materials should have a strong absorbing effect in military applications, particularly in an antiradar system. Thus, RAMs with wider absorption bandwidths and better absorption properties become more and more important (Wu et al., 2012). The transition metals and their oxides have a wide variety of applications. The carbonyl iron particles (CIP) and its compounds are good candidates for the electromagnetic interface shielding(Ahad et al., 2010). (Qing et al., 2010) investigated the microwave electromagnetic properties of carbonyl-iron particles as magnetic absorber and carbon fiber (CF) as conductive absorber filled insulating epoxy/silicone resin coatings. When the mass fraction of CIP was $65 \mathrm{wt} \%$ and CF was $2 \mathrm{wt} \%$, with coating thickness being $1 \mathrm{~mm}$, the reflectivity less than $-10 \mathrm{~dB}$ can be obtained in the frequency range of $8-18 \mathrm{GHz}$. (Ren et al., 2012)investigated the influence of FeCoB-based magnetic film on the absorption properties of traditional frequency selective surface. It is found that after the magnetic film is incorporated, the bandwidth under $-10 \mathrm{~dB}$ increases by $33.3 \%$ from 5.08 to $6.78 \mathrm{GHz}$ and the peak value of reflectivity decreases from $-12.46 \mathrm{~dB}$ to $-38.41 \mathrm{~dB}$. (Iwamoto et al., 2012) fabricated epitaxial thin films of Ru-substituted $\mathrm{CoFe}_{2} \mathrm{O}_{4}$ with a cubic spinel structure. The films showed room temperature ferrimagnetic behavior with hard magnetic features similar to those of non-Ru-substituted cobalt ferrite films. Both CIP and Co-ferrite particles are good candidates for fabricating RAMs. Hence the electromagnetic absorbing ability based on CIP and Co-ferrite filled in aliphatic polyurethane (APU) matrix was studied in this work. We studied their morphological by Field Emission Scanning electron microscopy (FE-SEM) and their electromagnetic parameters by S-Parameter Network Analyzer 8722ES. Their microwave absorbing characteristics were studied by Agilent PNA-X Network analyzer N5244A.

\section{Experimental Work}

\subsection{Materials and Fabricating of Coating Specimens}

We used Co-ferrite particles and CIP filled aliphatic polyurethane resin to fabricate electromagnetic absorbing 
coatings. Co-ferrite and commercial-grade CIP used in this work were brought from Tian Yi company. The CIP are spherical particles of around $2-5 \mu \mathrm{m}$ in diameter. The Co-ferrite particles embody variable shapes like rod, flake and sphericity. The specimens of $1 \#, 2 \#, 3 \#$, and $4 \#$ contained concentration of $0 \mathrm{wt} \%, 20 \mathrm{wt} \%, 40 \mathrm{wt} \%$, and $60 \mathrm{wt} \%$ Co-ferrite particles, and $60 \mathrm{wt} \%, 40 \mathrm{wt} \%, 20 \mathrm{wt} \%$, and $0 \mathrm{wt} \%$ CIP, respectively. The Co-ferrite particles and carbonyl iron powder separately distributed in acetone solution, with the method of ultrasonic bath, at room temperature for 15 minutes. Subsequently, Co-ferrite particles and carbonyl iron powder solution were mixed together by stirring for 20 minutes at $3600 \mathrm{rpm}$. Then the toluene, titanate coupling agent and dispersant were added to the mixture, stirring for 10 minutes at $1500 \mathrm{rpm}$. After aliphatic polyurethane resin had been added to the mixture, the compound substances were stirred uniformly at $2000 \mathrm{rpm}$ for 30 minutes at room temperature.

Table 1. Compositions and characters of coatings

\begin{tabular}{ccccc}
\hline Specimens & $\begin{array}{c}\text { Co-Ferrite particles } \\
\text { concentration }(\mathrm{wt} \%)\end{array}$ & $\begin{array}{c}\text { Carbonyl iron particles } \\
\text { concentration }(\mathrm{wt} \%)\end{array}$ & $\begin{array}{c}\text { Thickness of } \\
\text { coating }(\mathrm{mm})\end{array}$ & $\begin{array}{c}\text { Surface density of } \\
\text { coating }\left(\mathrm{kg} / \mathrm{m}^{2}\right)\end{array}$ \\
\hline $1 \#$ & $0 \%$ & $60 \%$ & 1.22 & 2.73 \\
$2 \#$ & $20 \%$ & $40 \%$ & 1.20 & 2.70 \\
$3 \#$ & $40 \%$ & $20 \%$ & 1.18 & 2.66 \\
$4 \#$ & $60 \%$ & $0 \%$ & 1.20 & 2.68 \\
\hline
\end{tabular}

After the fillers were well dispersed, the mixtures were sprayed on a flat aluminum plate $(300 * 300 * 2 \mathrm{~mm})$ by spray gun and kept in oven at $70{ }^{\circ} \mathrm{C}$ for complete drying. The spraying and drying procedures were repeated for four times. The thickness of specimens prepared was maintained to approximately $1.2 \mathrm{~mm}$.

\subsection{Characterization}

The morphologies of the specimens about CIP and (or) Co-ferrite particles were observed by a Hitachi S-4800 FE-SEM, operated at $15.0 \mathrm{kV}$ and the coating were observed at $1.0 \mathrm{kV}$. Cylindrical toroidal samples $(3.04 \mathrm{~mm}$ in diameter, $7.0 \mathrm{~mm}$ external diameter and $2 \mathrm{~mm}$ in thickness) were prepared by mixing paraffin. The weight ratio of absorbents was $40 \%$. And then the mixtures with absorbent and wax were pressed into the cylindrical compacts. We used transmission / reflection method to determine the relative permeability and permittivity via an HP-8722ES vector network analyzer system.

For the microwave absorbing characterization of the specimens, reflection/absorption measurements were carried out in the range of 4-18 GHz with the Naval Research Laboratory (NRL) arch method (Skolnik et al., 1970).

\section{Results and Discussion}

\subsection{Morphological Study}

Figure 1 shows the microstructures of CIP and Co-ferrite particles and profiles about $1 \#$ and $3 \#$ coatings. The images represents that the CIP and Co-ferrite particles were uniformly dispersed in the aliphatic polyurethane matrix.

The electromagnetic wave absorbing coatings can be fabricated with two different fillers successfully. CIP has spherical structure and Co-ferrite particles showing its spherical, flake, and rod shapes in the FE-SEM images. Dispersion of CIP and Co-ferrite particles was confirmed by their FE-SEM images. 

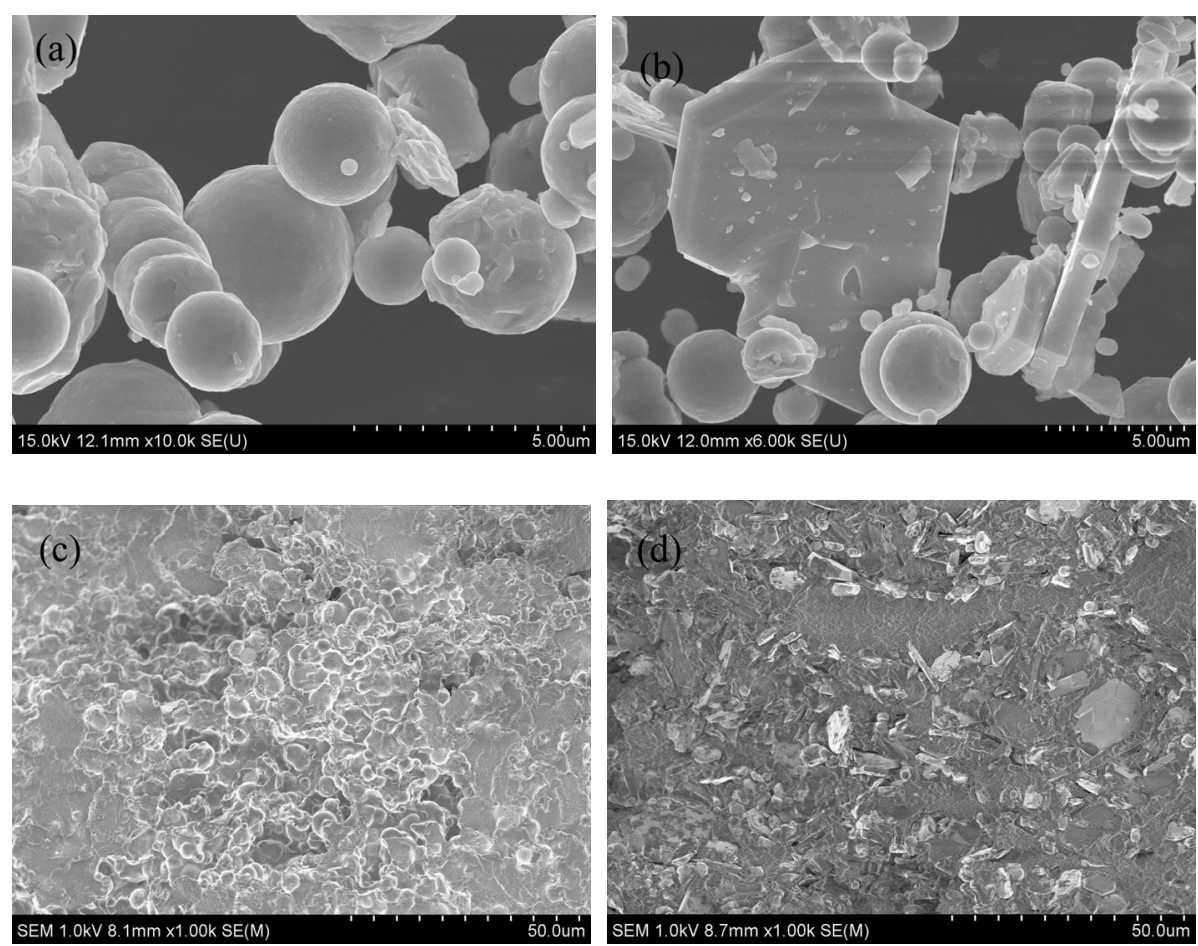

Figure 1. SEM images of $1 \#$ and $3 \#$ specimens

\subsection{Relative Complex Permittivity and Permeability}

A material's dielectric and magnetic properties are due to their complex permittivity and permeability parameters. And those of the CIP and Co-ferrite particles were measured to investigate the intrinsic reasons for electromagnetic wave absorption of the coatings by using the T/R coaxial line method. The complex permittivity is simply expressed by $\varepsilon=\varepsilon^{\prime}-j \varepsilon^{\prime \prime}$ in microwave frequency band, and the complex permeability is expressed by $\mu=\mu^{\prime}-j \mu^{\prime \prime}$. The real part of complex permittivity $\left(\varepsilon^{\prime}\right)$ represents the energy storage capacity. The imaginary part $\left(\varepsilon^{\prime \prime}\right)$ of the complex permittivity and permeability $\left(\mu^{\prime \prime}\right)$ are responsible for the energy loss mechanisms in the materials (Qing et al., 2010). The dielectric loss tangent of the mixture is expressed by $\operatorname{tg} \delta_{e}=\varepsilon^{\prime \prime} / \varepsilon^{\prime}$, and the magnetic loss tangent is expressed by $\operatorname{tg} \delta_{m}=\mu^{\prime \prime} / \mu^{\prime}$. The $\varepsilon^{\prime}, \varepsilon^{\prime \prime}, \mu^{\prime}$ and $\mu^{\prime \prime}$ of CIP used in the specimen are showed in Figure 2(a). The loss tangent $\left(\operatorname{tg} \delta_{e}\right.$ and $\left.\operatorname{tg} \delta_{m}\right)$ of CIP are showed in Figure 2(b).
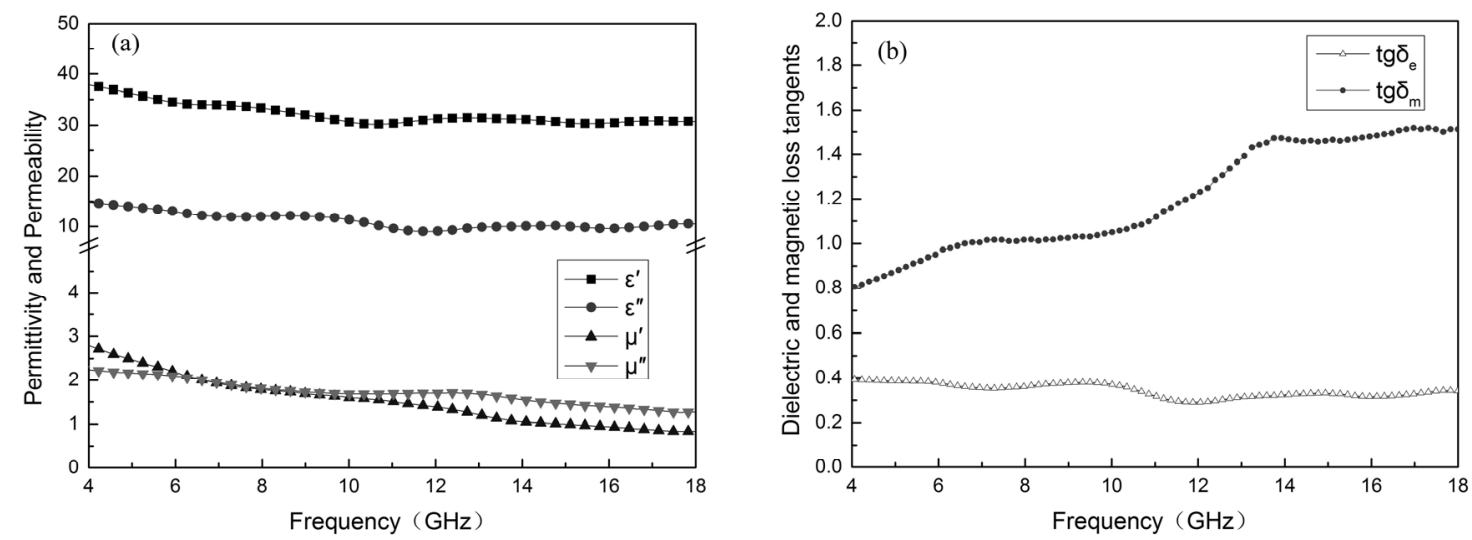

Figure 2. (a) Relative permittivity and permeability and (b) loss tangent of CIP 
The imaginary part of permittivity $\left(\varepsilon^{\prime \prime}\right)$ is around $9.03-14.83$ in the range $4 \mathrm{GHz}-18 \mathrm{GHz}$. And the imaginary part of permeability $\left(\mu^{*}\right)$ is around 1.27-2.24. The dielectric loss tangent of CIP almost kept constant with the frequency increasing, and the values of magnetic loss tangent increased with increasing frequency. Figure 3 represents the electromagnetic parameters $\left(\varepsilon^{\prime}, \varepsilon^{*}, \mu^{\prime}\right.$ and $\left.\mu^{*}\right)$ and loss tangent of Co-ferrite particles filled in wax, with wax concentration being $40 \mathrm{wt} \%$.
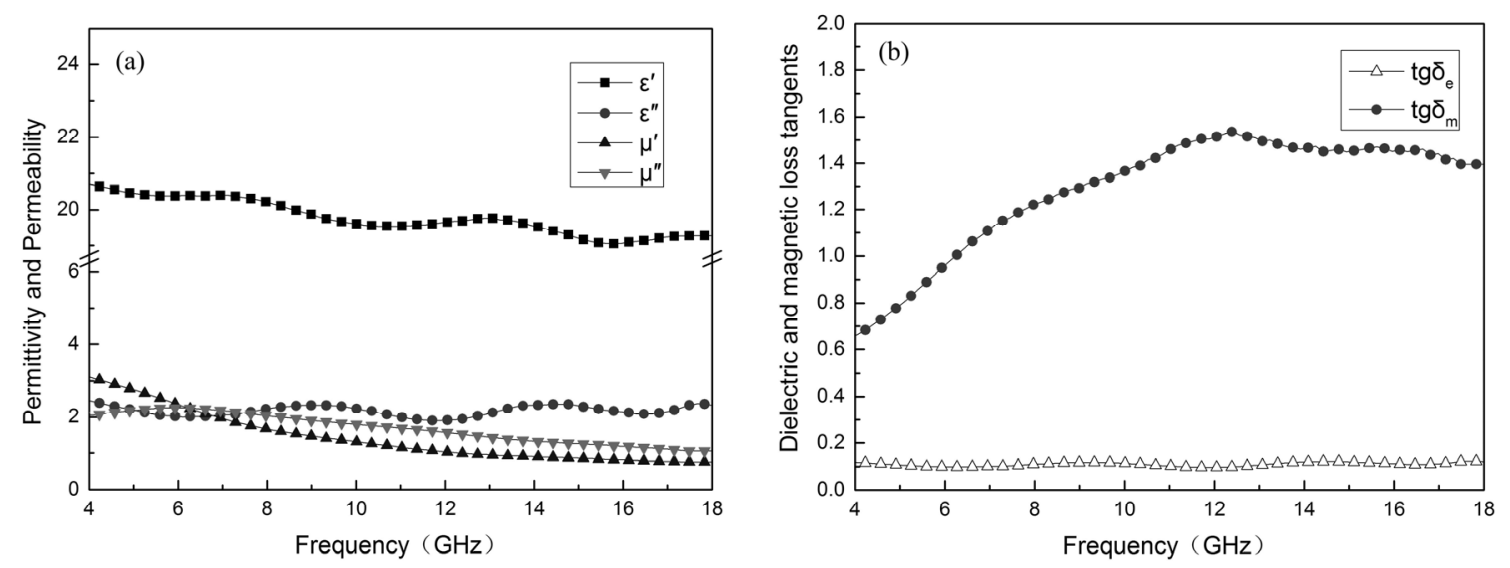

Figure 3. (a) Relative permittivity and permeability and (b) loss tangent of Co-ferrite particles

The imaginary part of permittivity $\left(\varepsilon^{\prime \prime}\right)$ of Co-ferrite particles is around $1.90-2.44$, far less than that of CIP. Meantime, the dielectric loss tangent is around 0.09-0.15, also less than that of CIP. It means that the dielectric lossy ability of CIP is superior to Co-ferrite particles in the range of $4 \mathrm{GHz}-18 \mathrm{GHz}$. The numerical values of complex permeability of both particles decreased with increasing frequency. The magnetic loss tangent of CIP increased sharply from 0.8 to 1.52 with frequency increasing from 4 to $18 \mathrm{GHz}$. And that of Co-ferrite particles increased slowly from 0.66 to 1.54 . It means that both CIP and Co-ferrite particle had certain ability of magnetic loss. Furthermore, the values of loss tangent $\left(\operatorname{tg} \delta_{e}\right.$ and $\left.\operatorname{tg} \delta_{m}\right)$ of CIP are more closer than those of Co-ferrite particles in the range of 4-12 GHz, which means that the values of them are more close to general matching law in designing absorbing materials in that range (Zhang et al., 2003).

Effective media theory is to calculate the effective electromagnetic parameters of several composite absorbing agents under different volume ratio and another absorbing material which is mixed by absorbing agent and penetrating agent, and analyze the absorbing properties (Zeng et al., 2011). On the condition that there are no large differences among the values of electromagnetic parameters of various components of the composite, the effective electromagnetic parameters are often calculated by the mixed formula:

$$
\varepsilon=\sum_{i=1}^{N} \varepsilon_{i} V_{i}
$$

Where $V_{i}$ is the volume proportion of the $i$ th component, and $\varepsilon_{i}$ is the permittivity and permeability. The mixed formula was used to calculate the parameters of the hybrid of CIP and Co-ferrite particles. As shown in Fig.4, the real part and the imaginary part of the permittivity are drift to the intermediate value with different proportion. But smaller changes in the real part and imaginary part of relative permeability can be seen. Therefore, the variation of reflection loss is mainly due to the variation of the relative permittivity after using the hybrid of CIP and Co-ferrite particles. 

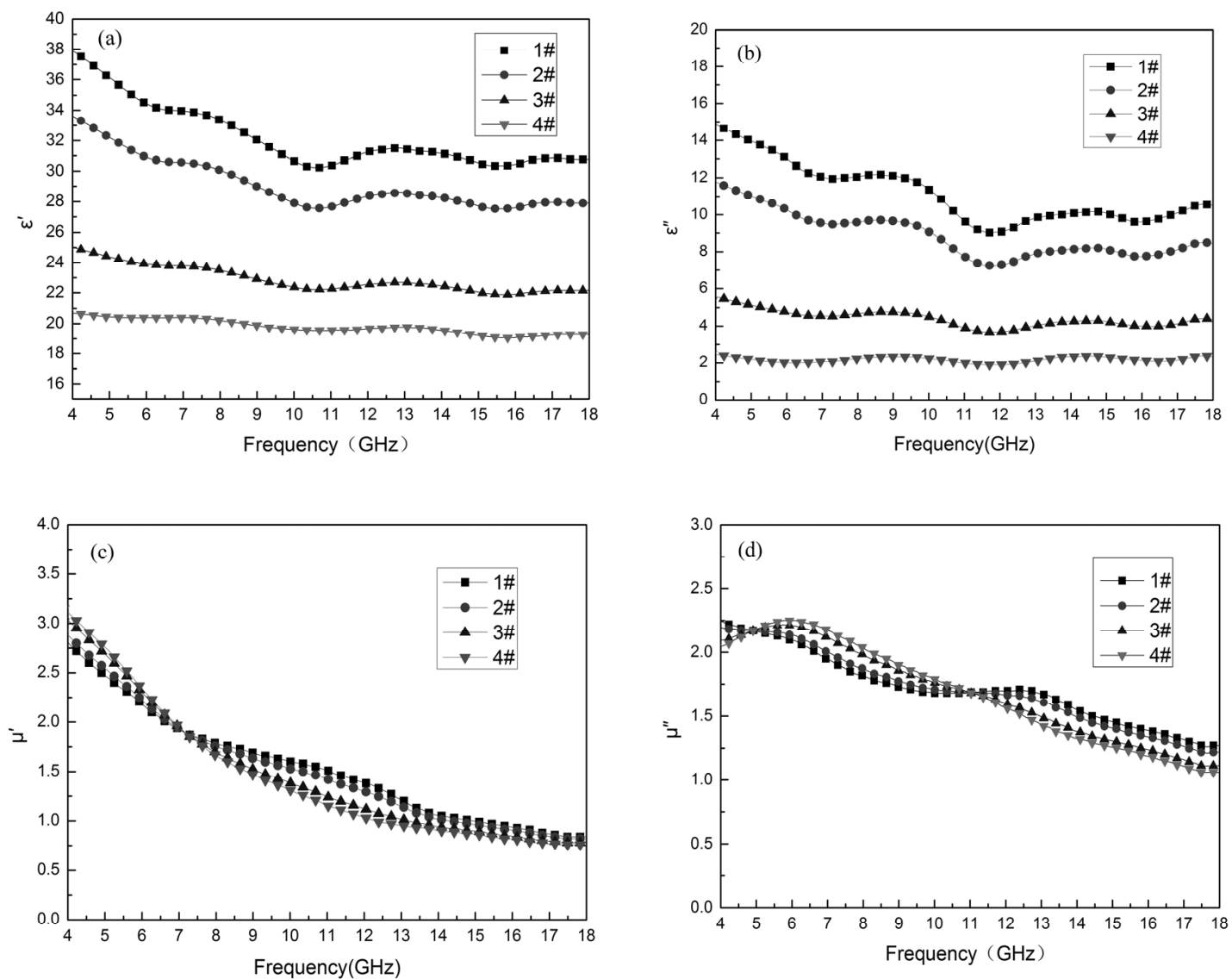

Figure 4. (a) Real part and (b) imaginary part of permittivity, (c) real part and (d) imaginary part of permeability of hybrid particles used in specimen $1 \#, 2 \#, 3 \#$ and $4 \#$, respectively

\subsection{Microwave Absorbing Properties}

The reflection loss (electromagnetic absorption) of the specimens was measured with NRL arch method and the results were shown in Figure 5. It expresses the relationship between reflection loss and frequency for the coatings with different CIP and Co-ferrite content.

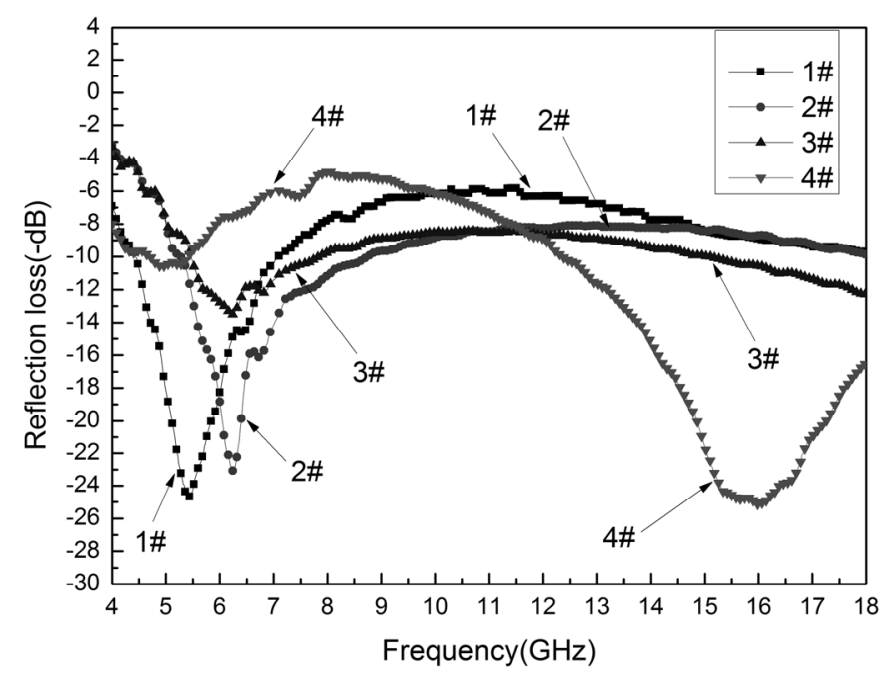

Figure 5. Reflection loss of the coatings with different content of CIP and Co-ferrite particles 
The specimens of $1 \#$ and $4 \#$ contained concentration of $60 \mathrm{wt} \%$ CIP and $60 \mathrm{wt} \%$ Co-ferrite particles, have RL values below $-8 \mathrm{~dB}$ in the range of $4-8 \mathrm{GHz}$ and $12-18 \mathrm{GHz}$, respectively. The minimum RL value of specimen $1 \#$ reaches $-24.77 \mathrm{~dB}$ at the frequency $5.42 \mathrm{GHz}$, and that of $4 \#$ reaches $-25.14 \mathrm{~dB}$ at $16.04 \mathrm{GHz}$. The effective bandwidths ( $\mathrm{RL}<-8 \mathrm{~dB}$ ) of $1 \#$ and $4 \#$ are $7.1 \mathrm{GHz}$ and $8.5 \mathrm{GHz}$. The ratio of CIP and Co-ferrite particles in the specimens $2 \#$ and $3 \#$ is $2: 1$ and 1:2 respectively. And the effective bandwidths of $2 \#$ and $3 \#$ are $12.9 \mathrm{GHz}$ and 13.0 GHz. It can be found that when the CIP (1\#) or Co-ferrite particles (4\#) used as main absorbents alone, a certain frequency band with good absorbing effect can be obtained. But the effective absorbing region is relatively narrow, with the characteristics of "narrow-band" features. When the CIP and Co-ferrite particles are mixed to be absorbents with certain ratio, the effective bandwidths of the absorbing coating is significantly increased.

Furthermore, the trend can also be seen that in the frequency band below $11.5 \mathrm{GHz}$, with the content of Co-ferrite particles increasing, the RL values of specimens (1\#, 2\#, 3\# and 4\#) increases. The minimum reflection loss moves to high frequency firstly, and then turns to low frequency. In the frequency band higher than $11.5 \mathrm{GHz}$, with the increasing of Co-ferrite particles content, the RL values of the coatings is decreasing sharply. According to the analysis of electromagnetic parameters, considering that the complex permittivity of the CIP is significantly higher than that of Co-ferrite particles, the mixed particles complex permittivity decreases compare to CIP relatively with the increasing of Co-ferrite content. At high frequencies (12 GHz-18 $\mathrm{GHz}$ ), with the continuous reduction of the content of CIP, the permittivity of the mixed particles constantly becomes smaller, and its value is closer to the relative permeability, which is conductive to the impedance match between the coating and free space, resulting in reducing the electromagnetic wave reflection.

\section{Conclusion}

CIP and Co-ferrite particles were used to fabricate electromagnetic wave absorbing coatings by traditional technology, with thickness been approximate $1.2 \mathrm{~mm}$ and the surface density about $2.7 \mathrm{~kg} / \mathrm{m}^{2}$. The minimum reflection loss of the specimens with hybrid particles is less than that of samples with CIP or Co-ferrite particles alone. But the effective absorption bandwidth $(<-8 \mathrm{~dB})$ can be significantly extended, increasing from about 7 $\mathrm{GHz}$ to $13 \mathrm{GHz}$. It is an effective method to broaden the effective absorbing bandwidth by mixing Co-ferrite particles and CIP with a certain ratio, meeting the requirements of broadband RAMs.

\section{References}

Ahad, F. B. A., Lee, S. F., Hung, D. S., Yao, Y. D., \& Yang, R. B. (2010). Magnetoelectric behavior of carbonyl iron mixed $\mathrm{Mn}$ oxide-coated ferrite nanoparticles. J. Appl. Phys., 107, 09D904. http://dx.doi.org/10.1063/1.3360357

Das, C. K., Bhattacharya, P., \& Kalra, S. S. (2012). Graphene and MWCNT Potential Candidate for Microwave Absorbing Materials. Journal of Materials Science Research, 1(2), 126-132. http://dx.doi.org/10.5539/jmsr.v1n2p126

Iwamoto, F., Seki, M., \& Tabata, H. (2012). Magnetic and electric properties of Ru-substituted $\mathrm{CoFe}_{2} \mathrm{O}_{4}$ thin films. Journal of Applied Physics, 112, 103901. http://dx.doi.org/10.1063/1.4766410

Qing, Y. C., Zhou, W. C., Jia, S., Luo, F., \& Zhu, D. M. (2010). Electromagnetic and microwave absorption properties of carbonyl iron and carbon fiber filled epoxy silicone resin coatings. Appl Phys A: Materials Science \& Processing, 100(4), 1177-1181. http://dx.doi.org/10.1007/s00339-010-5738-5

Ren, W. Y., Xiong, Y. N., Zhang. C., \& Zhou, Y. (2012). Enhancing and broadening absorption properties of frequency selective surfaces absorbers using FeCoB-based thin film. Journal of Applied Physics, 111, 07E703. http://dx.doi.org/10.1063/1.3670980

Skolnik, M. I. (1970). Radar Handbook. New York, NY: McGraw-Hill Book Company.

Wu, H. J., Wang, L., Guo, S., \& Shen, Z. (2012). Double-layer structural design of dielectric ordered mesoporous carbonparaffin composites for microwave absorption. Applied Physics A: Materials Science \$ Processing, 439-446. http://dx.doi.org/10.1007/s00339-012-6906-6

Zeng, C., Wu, C., Lv, X., \& Jia, Q. (2011). Analysis of Performance of Absorbing Materials Based on EMT. Safety $\$ E M C, 4,73-75$.

Zhang, H., Zhou, Z., Qin, B., Wang, D., \& Sun, H. (2003). Application of General Matching Law in design of multi-layer absorbing materials. Journal of Harbin Institute of Technology, 35(9), 1140-1143. 